\title{
Segmentation and Tracking of Carotid Artery from Ultra Sound Video Sequence
}

\author{
Syeda Nayeemunnisa ${ }^{1}$, Prof. Hussain Ahmed ${ }^{2}$ \\ $2^{\text {nd }}$ year, M.Tech, Electronics, H.K.B.K College of Engineering, Bengaluru, India ${ }^{1}$ \\ Head of Department, Electronics, H.K.B.K College of Engineering, Bengaluru, India ${ }^{2}$
}

\begin{abstract}
The accurate measurement of the lumen intima and media adventitia interfaces thickness is very important to find any kind of cardio vascular diseases that are progressing and present in the carotid artery, this can be achieved robustly by measuring and identifying the intima and media thickness, which also represents any kind of future cardio vascular diseases. Here we try to develop an algorithm that will automatically help in the analysis of the carotid artery by segmentation and tracking of the arterial thickness that will consider the B-mode video sequence of the ultra sound scan. In the first stage of the proposed algorithm the tracking and segmentation procedure is done and in the second stage a new technique called adaptive normalization correlation procedure is applied which helps the next coming frames to automatically adapt to the reference frames. This procedure is applied for one full cardiac cycle, the proposed algorithm using the automatic segmentation and tracking of the intima and adventitia thickness uses the adaptive normalization correlation technique is of very high clinical relevance importance and will provides the expected accurate result.
\end{abstract}

Keywords: IMT, CAD, ROI, IMC.

\section{INTRODUCTION}

Many heart and brain related diseases such as heart attack mode ultrasound scan is conducted which gives the clear whose scientific name is myocardial infarcts, predictor of the carotid artery. There will be nearly 35 to cerebrovascular events commonly known as strokes and 36 frames present in an ultrasound video sequence for one peripheral vascular disease also called as gangrene and cardiac cycle that is to be considered in order to take the kidney related diseases are most common around the local mean value for the measurement of the IMT. The world. All these diseases are caused due to the thickening normal IMT thickness should not be higher than 0.9 to of the carotid artery which is the largest artery and its $1 \mathrm{~mm}$ [1], if the IMT thickness is more than the reference pulse can be felt on either side of the neck under the jaw range then carotid artery is considered to have plaque line. The thickening of this artery wall results from formation and the physician can early diagnose and treat cholesterol, calcium and fibrous tissues caused by the myocardial infarctions. The ultra sound frame of a inflammatory-fibroproliferative responses due to rapid carotid artery is as shown in the fig. 1 .

lipid accumulation of many cells which are deposited in the form of plaque on the interior wall layer of the carotid artery. The carotid artery has three layers they are, the outer most layer is the Intimal layer followed by Media layer and Adventitia layer, and usually the plaque formation will be on the intima and median layer and thus we develop an automated algorithm for the segmentation and tracking of the intima and media thickness in the ultra sound video sequences of the common carotid artery, where we robustly identify and measure the intima media thickness which has high clinical references for identifying any cardio vascular events. And the IMT is best visualized in the longitudinal section of the common carotid artery wall [1].

Any kind of cardio vascular diseases can be found by finding the thickness of the IMT of the carotid artery that can be best visualized using ultrasound scan, usually a B-

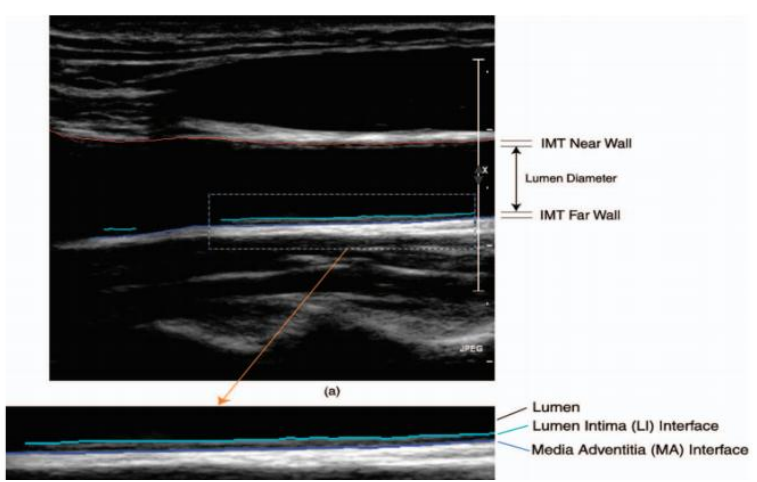

Fig.1. (a) First frame of the section of the carotid artery in the form of the longitudinal section (b) boundaries of the intima and media interfaces along with the lumen interface.

Advanced Image processing technique is used to provide an automated algorithm for the segmentation and tracking of the IMT, which is not restricted to one image but 
detects on the entire video sequence of the ultrasound scan of carotid artery with continuous number of frames, here a new technique called ANC that is Adaptive normalized correlation technique is used which will automatically adapt to the reference frame. The proposed algorithm will not only detect the existence of the plaque over the artery wall but it also indicates the existence of earlier cardio vascular disease that can be ruled by analyzing the elasticity or stiffness of the artery wall using mathematical relationships between the wall thickness and artery diameter were used to calculate the diameter enlargement on the thickness of the arterial wall by equating the wall areas of the artery on both the sides. The IMT for the diameter enlargement is not the diameter change of the linear function.

The computer aided diagnostic tool are more reliable and have the criteria and potential for the accurate measurement of the artery layers which will effectively reduce the inter and intra variability that is the major drawback in case of manual evaluation, therefore the computer aided detection (CAD) tool is very important for the correct evaluation of the inter and intra variability of the artery wall that will help the clinical practitioners for significant and accurate tracing of the plaque formation over the far walls of the carotid artery for the proper investigation of the myocardial infraction, the plaque formation over the intima and adventitia layers is as shown in figure 2. The algorithm provided will automatically track and segment the carotid artery along with the adaptive normalized correlation technique.

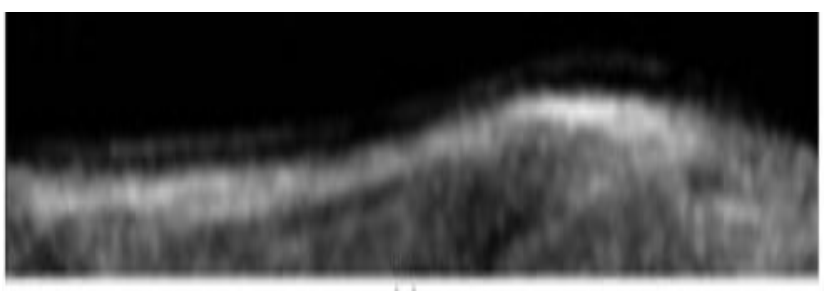

Fig.2. shows the carotid artery with the plaque formation where the bulky area is the plaque deposited over the artery wall.

The algorithm is carefully designed for the proper segmentation and tracking of the IMT that is tracked during the B-mode ultrasound video sequence, where the images are extracted from the ultrasound video sequence that are in the B-mode, which is one of the most know way that are commonly used in the mode of operations of the ultrasound equipment that are used by the clinical practitioners. The ultrasound images are the one which consist of scattered or reflected images and the sound is specified in the particular location generated from the sound waves, and the ultrasound videos are the real time videos in order to visualize the organs of the body and the ultra sound scan are the painless test that is conducted to create images or videos of the specified organs of the human body by using the sound waves, where theses sound waves are not radiations and are harmless and there are two types of ultrasound scan that are used to visualize the carotid artery where blood flow is also visualized that is flowing through the artery. Doppler and duplex are the two types of ultra sound scans that are conducted over the human body. Ultra sound is a high frequency sound that can be detected and emitted by special machines and the high frequency sound cannot be heard and this high frequency ultrasound can freely travel through blood, fluid and soft tissues. When ultrasound is reflected or bounced back when it hits solid surfaces such as bones and solid values then the ultra sound will echo back strongly of varying strengths.

The working of the ultra sound scan is very simple where the practitioner will apply the lubricating jelly on the skin and rubs the probe over the skin where the lubricating jell helps in good contact of the probe with the examining part of the body, this ultrasound probe is connected to the ultrasound machine through a wire, the pulses of the ultra sound are sent to the human body through the probe and the ultra sound waves are echoed back from the examined part of the body and the echoes that are detected by the probe are sent to the ultra sound machine through the help of the probe. There is a monitor linked to the ultrasound machine which displays the examined structure of the body where the picture frames are displayed and the updated pictures will have continuous movement of the structure that are taken from different angles. The ultrasound scan video sequence does not resemble a normal video sequence because it has lot of disturbance that will be created in the ultrasound video due the tissue contraction and patient movement.

\section{PROPOSED TRACKING ALGORITHM}

The measurement of the interfaces that are lumen intima and media adventitia is of great importance for the analysis of the thickness of carotid artery, which is best visualized for one cardiac cycle of the heart by making use of the computer development tool. For the analysis of the two interfaces that are the lumen intima and media adventitia interfaces, the measurement of their complexes is very important which makes use of the computer aided development tool for analysing the vascular model that is considered for one full cardiac cycle that consist of several processing steps, which are continuous and are spatial in nature. Thus the proposed algorithm will provide good segmentation and accurate measurement of the intima media thickness along with the adaptive normalization correlation technique. The block diagram of the proposed algorithm is as shown in the figure. 3 . 
A comprehensive outline of the algorithm for the IMC segmentation and tracking is provided where the data flow that is among the constituent modules is exemplified. The algorithm industrialized to fragment the IMC in the first frame of the arrangement is completely unsubstantiated and includes the following processing steps they are: automatic region of interest (ROI) detection, multiresolution edge filtering, IMC model selection, and edge reconstruction [1]. For the pursuing stage of the algorithm, the subsequent media adventitia and lumen intima interfaces that are recognized in the first frame are used to modify the proposed adaptive normalized correlation technique uses the tracing procedure that is applied where the IMC interfaces are identified in the ultra sound video sequence reference frames. The media adventitia and lumen intima edges display large inter-frame operational confined with the ultra sound video sequence. The proposed adaptive normalization correlation procedure has been considered to implement longitudinal consistency throughout the inter-frame tracing process and analysis of each and every processing steps are done.

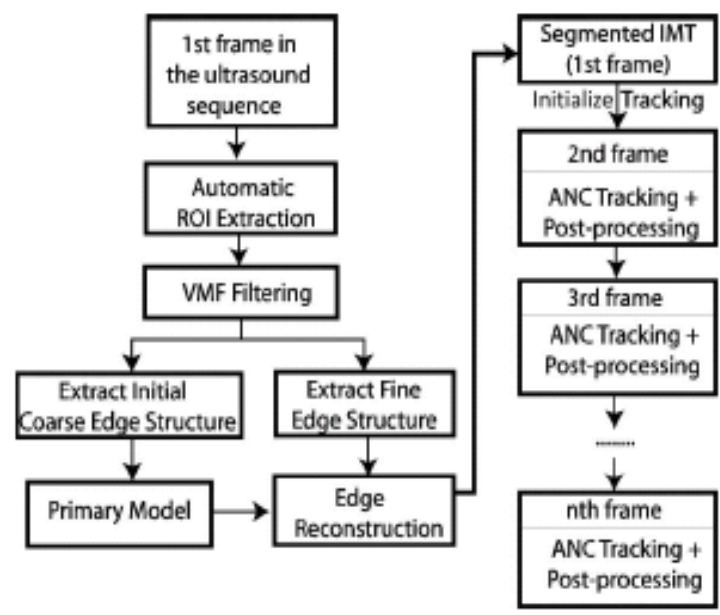

Figure.3.schematic representation of the proposed IMT segmentation algorithm.

\section{A. Contrast enhancement procedure}

For the functional structures of the frames of the arterial wall, if the anatomical pixels are low contrast pixels then the analysis of the frames will be difficult to analyse by the technician and it is one of the main disadvantage that is related to the ultra sound video sequence. If there are stumpy echo reactions that are caused by the ultrasound acquirement development, then affirmative sections of the IMT will have a compact divergence and they are not simply distinct from the other sections of the image frame of the arterial wall. For the improvement of the entrance of the IMT and simplify the recognition, a comprehensive contrast enhancement procedure is applied that is grounded on data broadening flanked by two pre-defined thresholds q min and q max, was useful[4].

$$
\mathrm{Vce}(\mathrm{X}, \mathrm{Y})=255 \mathrm{~V}(\mathrm{a}, \mathrm{b})-\mathrm{qmin}] / \mathrm{qmax}-\mathrm{qmin}
$$

The intensity value of the pixel $\mathrm{V}(\mathrm{a}, \mathrm{b})$ is situated at point $(a, b)$ in the image medium and the contrast enhanced intensity Vce $(a, b)$ value rate. Centred on the research q min and q max are the values that are fixed to 4 and 121 frames correspondingly and are retained unbroken for all pictures that are analysed in this learning. It is very important to notice the assortment of the two edges verified to be vigorous regardless of the ultrasound apparatus that has been programed to capture the image information from the carotid artery.

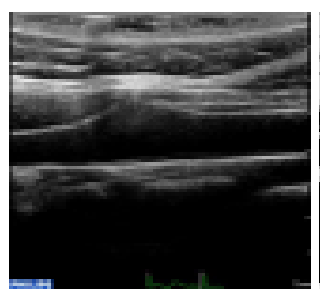

(a)

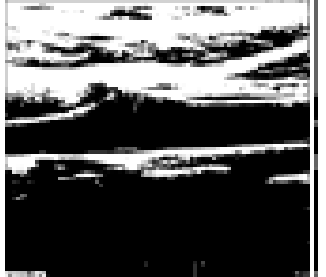

(b)
Figure.4. (a) original carotid ultra sound image (b) Image resulting from the contrast enhancement procedure.

\section{B. AUTOMATIC RECOGNISION OF AREA OF INTREST}

The exploration for the IMT that will be carried out in the area of interest, and it is the area that is positioned overhead and underneath the boundary where the blood and tissues of the arterial outer wall is detached. In order to avoid any human interference, we shall automatically identify the blood tissue boundary. The recognition of the tissue interfaces and vigorously fragment the two images of the blood and tissue an adaptive thresholding algorithm is applied in order to improve the image.

$$
f i=r i \div H, i \in[0,255], f i \geq 0, \sum_{i=0}^{255} f i=1
$$

From the above equation (2), the number $f i$ is the pixels within the grey level $\mathrm{i}$, and $\mathrm{H}$ is the total number of pixels in the image, $\mathrm{N}=\mathrm{n}_{0}+\mathrm{n}_{1}+\ldots+\mathrm{n}_{255}$ and ri represents the probability that the pixel with coordinates $(a, b)$ in the contrast enhanced image takes the value $\mathrm{i}$. The threshold $\mathrm{k}$ is automatically detected to maximize the between class variance [2]

$\max \in[0,255] \sigma(k)=\max \left\{\sum_{i=0}^{k-1} f i\left[\sum_{i=0}^{k-1}(i f i) / \sum_{i=0}^{k-1} f i-\sum_{i=0}^{255} i f i\right]\right.$
$+\sum_{i=k}^{255} f i\left[\sum_{i=k}^{255}(i f i) / \sum_{i=k}^{255} f i-\sum_{i=0}^{255}(i f i)\right]$

$$
\text { if } \mathrm{Vce}(\mathrm{a}, \mathrm{b})<\mathrm{k} \text { then } \mathrm{Vce}(\mathrm{a}, \mathrm{b})=0
$$




$$
\text { if } \operatorname{Vce}(\mathrm{a}, \mathrm{b})>\mathrm{k} \text { then } \operatorname{Vce}(\mathrm{a}, \mathrm{b})=255
$$

The modification technique is smeared to the picture consequential from the thresholding procedure is done with the determination of removing the trivial and false sections and achieves a additional condensed picture integration. The post handling process is approved out as monitored from the frames of the arterial wall of the ultra sound video sequence, from the each and every pixel in the thresholded picture a histogram $\mathrm{h} \Gamma(\mathrm{a}, \mathrm{b})$ is constructed in a $\Gamma$ locality. From the above equation, $s \max$ is a parameter that sets the typical deviation beyond max $b$ and inferior to $\min b$ to consent the attachment of supplementary evidence in the area of interest that will be essential in the procedure of IMT renovation. Investigations confirms that a $\mathrm{S} \max$ value is appropriately great to create a area of interest that concealments a bulky fragment of the lumen of the carotid artery further than the adventitia regardless of the perseverance of the ultrasound images. The divergence heightened image statistics experimented by the area of interest will be additional managed for the documentation of the intima media thickness.

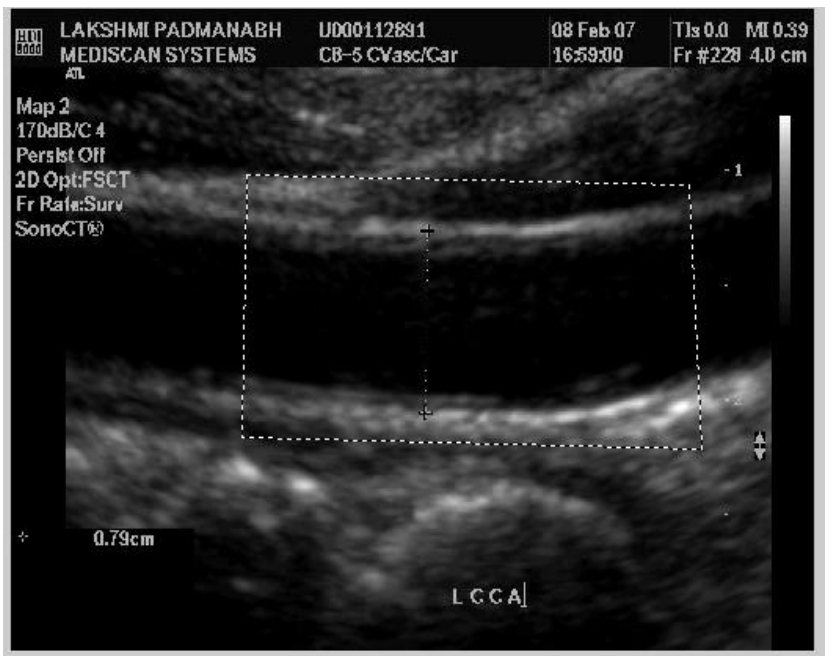

Figure.5. Snap shot of the carotid artery frame showing the selection of area of intrest.

\section{VMF FILTERING}

The type of filter that is used here is a Vector median filter that implements a nonlinear filtering operation, where a window moves over a signal and at each and every point of the median value of the data within the window is taken as the output. Median filtering has some incapacitate possessions that cannot be attained with linear algorithm [3]. The impulse response of the median filter is null. The nonlinear property of the filter makes its use striking in conquering imprudent noise. The vector Median filters are vigorous and are well matched for data crumpling when the noise physiognomies are not known. Several changes are made stepwise when a signal passes the median filter infrangible. This possession is used in the applications such as image filtering, and the data needs to be sharp but distorting of the signal boundaries is not satisfactory and it also eliminates all categories of clatter that is contemporary in the image of the ultra sound video sequence.

The median of $\mathrm{N}$ scalars $\mathrm{Zi}=1, \ldots, \mathrm{N}$, can be defined as the value $\mathrm{Z}$ med such that for all $\mathrm{y}$,

$$
\sum_{i=1}^{N}[Z \operatorname{mad}-Z i] \leq \sum_{i=1}^{N}[-Z i]
$$

We can always choose the $\mathrm{Z}$ med value as one of the $\mathrm{Zi}$. If $\mathrm{N}$ is anomalous, $\mathrm{Z}$ med is inimitable, and if $\mathrm{N}$ is uniform there can be an immeasurable number of options for $\mathrm{Z}$ med. For the vector median filters $\mathrm{N}$ is preferred to be anomalous. And if we assume that we have a unsystematic example $\{Z 1, \ldots, Z n\}$ from a inhabitants having a bi exponential thickness meaning. Vector median filters have been functional to both one and two dimensional pointer dispensation. And the presentation zones contain discourse dispensation. The application extents include speech processing and image improvement procedure. We have many analyzing arithmetical and derivation indicator properties of these median filters, and we have to familiarize some adjustments and simplifications for the median filters.

\section{INITIAL AND FINE COARSE EDGE STRUCTURES}

The subsequent stage of the procedure excerpts the initial edge and fine edge structure of the conceivable intima and media thickness that fragments about the area of importance using the Canny edge detector [9]. The chief motivation for choosing Canny for edge detection is that it accomplishes decent recognition, virtuous localization and also gratifies the one rejoinder average by reducing numerous reactions for a particular verge. The Canny edge detector excerpts the incline of the picture statistics that is paramount convolved with a two Dimentional Gaussian filter, the gaussian filter is used to decrease the existence of unauthentic boundaries instigated by noisy picture. The measure $\sigma$ is an important restriction that is used to set the extent of the Gaussian filter and the assessment is set in combination with the anticipated level of boundaries, one of the acceptable contrasted with coarse boundaries. Let us take the contemplation that fine boundaries are abundant and require an unequal that is twisted presence, and this also produces a problematic situation when the boundaries sections are examined in the procedure of the intima and media thickness recognition. In order to statement the problem, the procedure projected here implements a coarse to fine approach for the intima and media thickness recognition. The initian and coarse edge recognition is achieved with the help of gaussian filter where a scale of the Gaussian machinist is established in order to increase the superiority connectivity and eliminate the fragile boundaries, some of the non minimums destruction and thresholding are functional [4]. The assessment of the scale constraint confirms that the inappropriate boundaries resulting from the picture clatter and fragile surfaces are detached and only the resilient boundary structures are 
estimated to abstract the principal the intima and median thickness.

\section{E. RECONSTRUCTION OF THE DATA FRON EDGES}

The last step of the projected intima and media separation procedure encompasses the achievement of the coarse intima consuming the verge material that is achieved by smearing the VMF filtered data also by using canny's edge detection technique with a minor measure assessment. For this computational stride, the coarse intima media complex edges will be enlarged with the fine measurement boundaries fragments that are spatially adjoining and dependable with the geometrical and anatomic constrictions that were enforced in the creation of the initial and coarse intima and media complex.

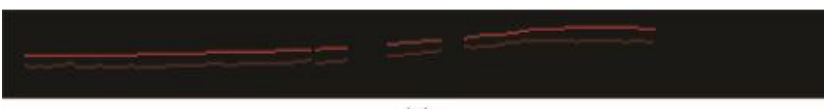

(a)

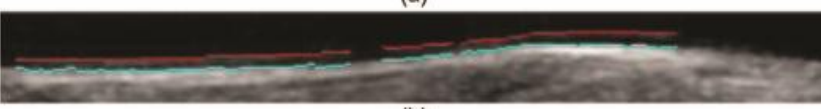

(b)

Figure.6. (a) The primary intima media complex (IMC) model (b) The concluding pair of lines of the IMC subsequent after the boundaries data

$X(p)$
$=\frac{\left[\sum_{(x, y) \in[1, n p]} \sum_{q \in(0,3]} I(a, b+N)\right]}{3 n p}$

$\begin{aligned} & Y(p) \\ = & \frac{\left[\sum_{(x, y) \in[I, n p]} \sum_{q \in(0,3]} I(a, b-N)\right]}{3 n p}\end{aligned}$

The enduring fine edge statistics comprise of a enormous quantity of boundary fragments, and only the contiguous are situated in the nearby locality of the coarse chief intima media complex can be reserved for additional investigation. In order to accomplish this, a gradient of boundary terminators is removed for every section of the principal, where the segments of the primary intima media complex, and the exploration for contender edge sections is introduced in contract with neighborhood and principal IMC symmetrical restrictions, which is accomplished by erecting an selection in which, for each characterized section of the principal intima media complex is achieved.

The projected pursuing procedure is approved out by estimating the adaptive normalization correlation technique along with all opinions that outlines the intima media complex that is consequential from the submission of the subdivision technique that has been delineated. The main benefit of the future ANC established tracing arrangement is that is not vulnerable by the nonappearance of robust inclines, and with the situations of presentation is established on vigorous delineation approaches. Figure.7. shows the complete output of the entire code that is running in order to obtain the thickness of the carotid artery from the ultra sound video sequence.

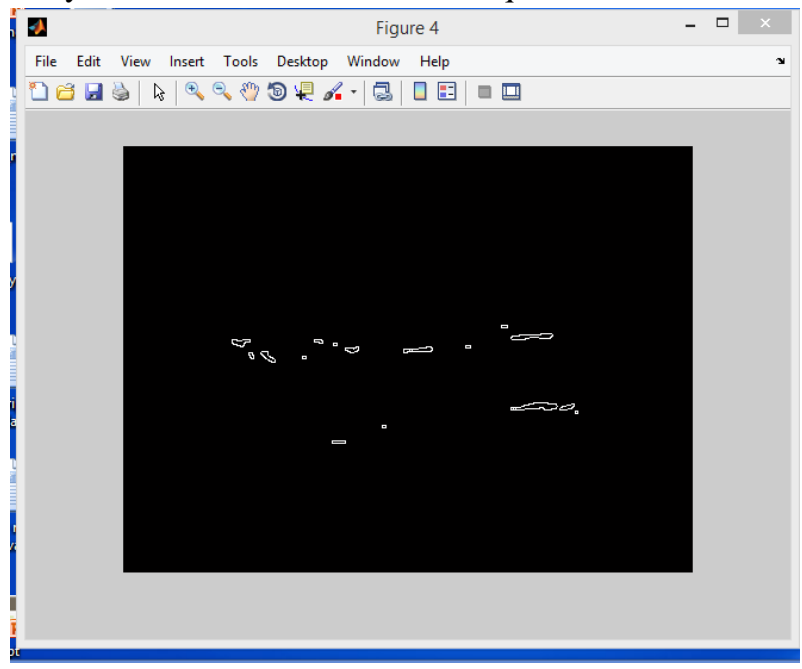

Figure.7. shows the snapshot of the carotid artery image after edge data reconstruction.

\section{CONCLUSTION}

The main objective of this paper was to familiarize a innovative algorithm for the dissection of the intima and media complex in the longitudinal section of ultra sound carotid artery pictures. The foremost innovation of this method exist in in the expansion of an unverified procedure that implants a arithmetical intima media complex that is modelled in a course to fine approach. The projected procedure verified to give correct dissection consequences when practically applied to numerous carotid ultrasound pictures that are considered by low tenacity and high level of image sound. This investigation is continuing and we strategy to lengthen the competences of the proposed ANC system to spontaneously measure the intima media complex in ultrasound carotid figures in order to allow the scheming of dynamical possessions of the carotid artery images.

\section{ACKNOWLEDGMENT}

I am very grateful and would like to thank my guide HOD. Prof. Hussain Ahmed for his advice and continued support. I would like to thank my husband Dr. shiraz pasha for his whelming and constant support and my friend for the thoughtful and mind stimulating discussion we had, which prompted us to think beyond the obvious.

\section{REFERENCES}

[1] Dana E. Ilea, Caoimhe Duffy, Liam Kavanagh, Alice Stanton, and Paul F. Whelan "Fully Automated Segmentation and Tracking of the Intima Media Thickness in Ultra sound Video Sequences of the Common Carotid Artery” IEEE 2013, vol. 60.

[2] Dana E. Ilea, Paul F, Whelan Senior and Alice Stanton An 2D CAD Algorithm for the Segmentation of the IMT in Ultra Sound Carotid Artery Images IEEE 2009, Minnesota, USA.

[3]J. Astola, P. Haavisto, and Y. Nuevo, "Vector median filters", Proc. of IEEE, vol. 79, pp. 678-689, 1990. 
INTERNATIONAL JOURNAL OF INNOVATIVE RESEARCH IN ELECTRICAL, ELECTRONICS, INSTRUMENTATION AND CONTROL ENGINEERING Vol. 3, Issue 5, May 2015

[4] J. Canny, "A computational approach to edge detection", IEEE Trans. on Pattern Analysis and Machine Intelligence, vol. 8, no. 6, pp. 679698, 1986.

[5] C. Liguori, A. Paolillo, and A. Pietrosanto, "An automatic measurement system for the evaluation of carotid intima-media thickness", IEEE Trans. on Instrumentation and Measurement, vol. 50, no. 6, pp. 1684-1691, Dec. 2001.

[6] R. H. Selzer, W. J. Mack, P. L. Lee, H. Kwong-Fu, H. N. Hodis, "Improved common carotid elasticity and intima-media thickness measurements from computer analysis of sequential ultrasound frames", Atherosclerosis, vol. 154, no. 1, pp. 185-193, 2001.

[7] F. Faita, V. Gemignani, E. Bianchini, C. Giannarelli, and M. Demi, "Real-time measurement system for the evaluation of the Intima Media Thickness with a new edge detector", in Proc. 28th IEEE. Engineering in Medicine and Biology Society, pp.715-718, 2006

[8] Q. Liang, I. Wendelhag, J. Wikstrand, and T. Gustavsson, "A multiscale dynamic programming procedure for boundary detection in ultrasonic artery images", IEEE Trans. on Medical Imaging, vol. 19, no. 2, pp. 127-142, Feb. 2000.

[9] C. P. Loizou , C. S. Pattichis , M. Pantziaris , T. Tyllis, and A. Nicolaides, "Snakes based segmentation of the common carotid artery intima media", Medical and Biological Engineering and Computing, vol. 45, no. 1, Jan. 2007.

[10] F. Destrempes, J. Meunier, M.-F Giroux, G. Soulez, and G. Cloutier, "Segmentation in Ultrasonic B-Mode Images of Healthy Carotid Arteries Using Mixtures of Nakagami Distributions and Stochastic Optimization", IEEE Trans. on Medical Imaging, vol. 28, no. 2, pp. 215-229, Feb. 2009.

[11] N. Otsu, "A threshold selection method from gray-level histograms", IEEE Trans. on System, Man, and Cybernetics, vol. 9, no. 1, Jan. 1979 